\title{
WOUND IMAGE ANALYSIS CLASSIFIER FOR EFFICIENT TRACKING OF WOUND HEALING STATUS
}

\author{
K. Sundeep Kumar ${ }^{1}$ and B. Eswara Reddy ${ }^{2}$ \\ ${ }^{1}$ Associate professor, Department of Information Science \& Engineering, \\ SEA CET, Bangalore, India \\ ${ }^{2}$ Professor, Department of Computer Science \& Engineering, \\ JNTUA College of Engineering, Anantapur, AndhraPradesh, India
}

\begin{abstract}
Wounds are evolved by increase in number of damage tissues. The traditional way of assessing the wound healing status is to periodic measure of the area covered by the wound. This technique is tedious to measure and periodic assessment is cumbersome. Basically healing status of the wound can be classified as contact methods and non contact methods. The purpose of this research work is to accurately assess the healing status of the wound .To accurately assess the wound, capturing of the wound images are the first task to be performed. There are various tools like the photographic wound assessment tool (PWAT) to acquire efficient wound images. Since the characteristics of different types of wounds (venous, pressure, diabetic, and arterial ulcers) vary markedly, determining the reliability and validity of using the PWAT to assess wound appearance for both chronic pressure ulcers and leg ulcers due to vascular insufficiency is important. Segmenting the area of the wound from the wound image using efficient segmentation techniques and preprocessing the segmented wound to reduce the noise using efficient filters and efficient denoising techniques. Efficient classifiers are needed to classify the wound images. One among the classifiers are the Wound Image Analysis Classifier (WIAC). Experimental evaluation has been made on comparing various classifiers like SVM, KNN, WIAC.
\end{abstract}

\section{KEYWORDS}

Skin, Wound types, Filtering, Segmentation, Classification

\section{INTRODUCTION}

An accurate and thorough wound assessment is an essential component of optimal wound care. A wound assessment serves two important purposes:1) to determine wound severity in order to predict expected rate of wound healing and develop a comprehensive plan of care and 2) to act as a reliable outcome measure that can be used to assess the effectiveness of a given wound treatment program. A key parameter that should be included in a wound assessment is the measurement of wound extent [5]. Several methods of determining wound size have been developed and validated including wound depth [3], surface area [6],[7] length and width [8] and volume[9]. These methods have been compared. And although each method of estimating wound size has inherent strengths and weaknesses, those used to determine wound surface area, rather

DOI : $10.5121 /$ sipij.2014.5202 
than wound depth or volume, are believed to be most accurate and reliable[8],[10-13]. A comparison of several different methods available to assess wound surface area has also been performed, and although stereophotographic determinations may be most accurate, the use of a wound tracing onto a transparent acetate or calculations made from length/width measurements are considered reliable and easy to use[12-15]. Other determinants of wound healing that should be included in a wound assessment are the evaluation of wound bioburden and wound severity [5].This requires examining wound exudate and necrotic tissue type, the amount and characteristics of necrotic tissue, granulation tissue, and reepithelialization, and assessing the viability of the wound edge and periulcer skin[16]. To assess these components of wound healing, several assessment tools have been developed including the pressure sore status tool (PSST) [12-13], the pressure ulcer scale for healing (PUSH Tool)[10], the Sussman wound healing tool (SWHT)[21] the Sessing Scale[22], and the wound healing scale (WHS)[13]. Recent reviews of these wound status measurement tools found that at least two of these scales (PSST and Sessing Scale) have sufficient published information to be considered valid and reliable measures of wound healing in chronic pressure ulcers [14],[15]. All of these wound status tools or scales require that an expert healthcare professional who is trained in wound evaluation perform a bedside assessment of the wound bed, wound edge, and periulcer skin and assign a number to the wound that best describes the observations made. All of the instruments currently available to assess wound status (except for the WHS) were developed specifically for use on pressure ulcers. None have been shown to accurately assess the appearance of chronic vascular leg ulcers such as diabetic, venous, or arterial ulcers.

A recent survey of home healthcare agencies in the United States revealed that $75 \%$ of agencies included a wound photograph as part of the documentation procedures. Additionally, numerous publications that describe how to perform a wound evaluation advocate the use of wound photographs to capture wound status. Using wound photographs has some major advantages including the fact that photographs do not require that the provider come into contact with the wound. Photographs also identify the physical dimensions of the ulcer as well as the type of tissue present within the wound bed. According to Hughes, "A medical photograph can transfer more information to a health professional than subjective descriptions, which are open to misinterpretation." In one reported case, photodocumentation was useful in obtaining medical reimbursement for a patient. Several new types of automated and computerized technologies that capture and analyze photographic images of chronic wounds have been developed to assist the clinician with wound healing documentation. Furthermore, visual images of wounds could be used to electronically transmit information about healing status to a remote location for consultation by a wound care expert. This application of telemedicine has been used to facilitate optimal wound care in patients who have chronic wounds and are not located in major health centers.32 To date, as many as $83 \%$ of dermatologic conditions have been accurately diagnosed using telemedicine services.33 Recently, Wirthlin et al34 demonstrated that physicians viewing a wound image using computerized imaging technology were able to determine the presence or absence of wound descriptors and showed agreement on basic wound management decisions such as whether the patient required further investigation. Despite the relatively frequent use of wound photographs to document wound healing, it is unknown whether these visual images of the wound can be used to provide a valid and reliable assessment of wound status.

The purpose of this research work is to accurately assess the healing status of the wound .To accurately assess the wound, capturing of the wound images are the first task to be performed. There are various tools like the photographic wound assessment tool (PWAT) to acquire efficient 
Signal \& Image Processing : An International Journal (SIPIJ) Vol.5, No.2, April 2014

wound images. Since the characteristics of different types of wounds (venous, pressure, diabetic, and arterial ulcers) vary markedly, determining the reliability and validity of using the PWAT to assess wound appearance for both chronic pressure ulcers and leg ulcers due to vascular insufficiency is important. Efficient classifiers are needed to classify the wound images. One among the classifiers are the Wound Image Analysis Classifier (WIAC).

\section{WOUND ANALYSIS}

\subsection{Wound Assessment}

Accurate wound assessment is essential to the appropriate and realistic planning of goals and interventions for patients with wounds. However, the assessment process has a number of components that must be systematically considered

The process of wound assessment requires a range of skills and knowledge including:

- Knowledge of relevant anatomy and physiology;

- The ability to identify factors that may interfere with normal wound healing;

- The ability to collect objective and subjective data;

- The ability to analyse and interpret the information that has been gained;

- The ability to identify the patient's problems and needs through discussion.

Components of the assessment process

When assessing a patient with a wound, four questions need to be considered:

- What is the aetiology and location of the wound?

- How should the wound be graded using an objective grading tool?

- Based on the wound grading, what is the primary treatment objective?

- What regimen is required to achieve the identified treatment objectives (Collier, 2000)?

The following 10-point guide provides a systematic approach to wound assessment.

\section{Classification of the wound}

There are four main groups of wounds.

$>$ Mechanical - for example surgical and traumatic wounds.

$>$ Chronic - for example leg ulcers and pressure ulcers.

$>$ Burns, chemical or thermal injuries-these may be further classified by depth of injury.

$>$ Malignant - primary lesions such as melanomas.

Initially all wounds can be described as acute and are anticipated to progress through a normal wound-healing process. However, if they become fixed in one of the major phases of healing (inflammation, regeneration or maturation) for a prolonged period - for example more than six weeks, the wound can be described as chronic. 
Signal \& Image Processing : An International Journal (SIPIJ) Vol.5, No.2, April 2014

\section{Information that assists wound assessment}

The assessment process should be holistic, systematic and evidence based. Some authors have suggested basing assessment on existing nursing models (Bale and Jones, 1997; Collier, 1994). The assessment framework described in this article should help you identify factors in three areas that may delay wound healing - general health, wound management and the local wound environment. General health includes age, social and care environments, psychological perspectives, nutrition, medical diagnosis and associated disease processes, medical interventions - for example drug therapy and history of the wound.

\section{Information required to assess a wound}

The assessment process, which can be assisted by the use of a wound-assessment tool, should help identify.

$>$ The number and location of wounds.

$>$ The grade of the wounds - colour and/or numerical grading tools may be used (Collier, 1994).

$>$ The size of the wound - dimensions (length and breadth) can be measured using a plastic ruler - which should be disinfected - or approximated from a tracing on a proprietary measuring grid.

$>$ The nature of any wound fluid - is it exudate, serous fluid or pus?

$>$ The cause, nature and severity of any pain related to the wound. There are many causes of wound pain, which may be determined using a pain assessment scale (Hollinworth and Collier, 2000).

\section{Additional techniques that can inform the assessment process}

The use of technology can often help to clarify both the aetiology and extent - which is often not visible - of a wound being assessed.

To assist with the differential diagnosis of leg ulcers the following may be incorporated into the assessment:

Doppler ultrasound.

$>$ Duplex scanning.

$>$ Photoplethysmography (PPG).

The following may assist with assessing the extent of pressure ulcers, sinuses and dehisced wounds:

$>$ Colour Doppler ultrasound.

$>$ Sinography.

$>$ Other investigations such as computerised tomography (CT) or magnetic resonance imaging (MRI).

Computer-based assessment systems can be used to assist the objective measurement of all wound types, as they can assess maximum dimensions of the wound plus depth, volume and are non-invasive. 
Signal \& Image Processing : An International Journal (SIPIJ) Vol.5, No.2, April 2014

\section{Assessment of the surrounding skin}

Assessment should identify whether the skin is erythematous, excoriated, indurated or macerated.

$>$ Erythema - redness of the skin as a result of a hyperaemic response - can be due to the presence of micro-organisms within the local tissues, which are not multiplying (colonisation), or micro-organisms, which are multiplying and causing an additional host reaction, for example a change in the amount/nature of wound fluid as a result of infection.

$>$ Excoriation - stripping of the upper layers of the epidermis as a result of prolonged exposure to toxins on the surface of the skin - can occur when there is excessive wound fluid.

$>$ Induration - a change in the texture rather than colour of the skin - it becomes hardened and less supple.

$>$ Maceration - excessive moisture in the skin resulting in the tissues becoming waterlogged.

\section{Identify primary treatment objectives}

These depend on the signs and symptoms identified in the assessment. Typical objectives may include:

$>$ Wound cleansing - does the wound need to be cleansed and if so, with what? It is generally accepted that wounds should be cleansed with normal saline, with the exception of some chronic wounds. Routine cleansing is not recommended for clean wounds.

$>$ Debridement/desloughing can be achieved using a number of methods including mechanical, for example, sharp debridement; autolytic, for example rehydration of the tissues; enzymatic; or biosurgery (larval therapy).

$>$ Control of exudates.

$>$ Control of bleeding.

$>$ Minimizing the effects of infection.

The objectives may involve more than one of the above, However, in all cases the prime objective should be to optimise the patient's healing potential.

\section{Interventions to achieve the planned outcome}

Interventions can include the use of: 
Signal \& Image Processing : An International Journal (SIPIJ) Vol.5, No.2, April 2014

$>$ Interactive wound management materials - these include alginates, enzymes, foams, films, hydrocolloids, hydrofibres and hydrogels that help to create or maintain the optimum healing environment (Collier, 1996).

$>$ Active wound management materials - these incorporate growth factors such as epidermal growth factor (EGF) or platelet-derived growth factor (PDGF) that stimulate the healing environment.

$>$ New technologies such as hyperbaric oxygen therapy, low level laser therapy and topical negative pressure.

$>$ Complementary therapies (it is important to understand the rationale for the use of these).

Once the primary treatment objective has been achieved, assessment should be repeated to identify the next objective and so on until wound closure.

\section{Documentation}

This is essential, both as a record of best practice at the time it was written and as a vital communication tool that should be accessible to all professionals involved in the management of the patient's wound. All components of the assessment process should be documented using unambiguous terminology. The use of formal wound assessment charts may be helpful as they can focus the assessment process.

\section{Photographic records}

Before any photographic record is obtained, it is important to identify its purpose. It could be argued that if the answer to all of the following questions is 'no', then photography is unnecessary:

$>$ Is it for record-keeping, highlighting the anticipated progress of wound healing?

$>$ Is it for inclusion in a case report file because the patient has been recruited into a clinical evaluation or research trial?

Is it for historical archiving because the wound is rare or unique in nature?

\section{Patient information}

All patients are individuals and will require different information during and after the process of assessing and managing the wound. This should be presented in an accessible form.

\subsubsection{Types of tissues in a wound:}

After considering the various factors involved in analyzing the wound healing process, there is a need of analyzing various types of tissues present in forming a wound . Types of tissues present in wound are as follows. 
Signal \& Image Processing : An International Journal (SIPIJ) Vol.5, No.2, April 2014

\begin{tabular}{|c|l|l|}
\hline Sl.NO. & \multicolumn{1}{|c|}{ Tissue } & \multicolumn{1}{c|}{ Description } \\
\hline 1 & Necrotic tissue & $\begin{array}{l}\text { Non viable tissue, dead no blood flow. Ex: Slough(Yellow, } \\
\text { green, gray, lighter, thin wet, stringy), Eschar (black, } \\
\text { brown, grey, darker, thicker, harder) }\end{array}$ \\
\hline 2 & Epithelial Tissue & $\begin{array}{l}\text { Outer most layer of the skin, deep pink to pearly pink, } \\
\text { close the wound }\end{array}$ \\
\hline 3 & Granulation Tissue & $\begin{array}{l}\text { New tissue that replaces the dead tissue. Beefy red, puffy, } \\
\text { mounded, grows from base of wound }\end{array}$ \\
\hline 4 & Hyper Granulaion tissue & Forms above surface. Delays epitheliazation \\
\hline 5 & Muscle tissue & $\begin{array}{l}\text { Pink to dark red. Highly vascularized (richly supplied with } \\
\text { blood), Striated(striped, grooved or ridged) }\end{array}$ \\
\hline 6 & Tendon & Attaches muscle to bone. Shiny when healthy \\
\hline 7 & Fascia & Covering over muscles. Shiny, white \\
\hline 8 & Bone & Shiny, smooth \\
\hline
\end{tabular}

Table 1 Types of tissues

Color description of various types of tissues involved in forming a wound. After identifying the intensity of various colors involved in a wound by calculating color histogram. Healthy tissues and unhealthy tissues classification can be made easy. The following table describes color and description about the color in analyzing the wound.

\begin{tabular}{|c|l|l|}
\hline Sl. No. & $\begin{array}{c}\text { Wound } \\
\text { Base color }\end{array}$ & \multicolumn{1}{c|}{ Description } \\
\hline 1 & Beefy red & Healthy tissue, good blood flow \\
\hline 2 & Pale pink & Poor blood flow, anemia \\
\hline 3 & Purple & $\begin{array}{l}\text { Engorged, swelling, high bacteria } \\
\text { levels, trauma }\end{array}$ \\
\hline 4 & Black & Non viable necrotic tissue \\
\hline 5 & Brown & Non viable necrotic tissue \\
\hline 6 & Yellow & Non viable tissue, slough \\
\hline 7 & Green & Infection, non viable tissue \\
\hline 8 & White & Macerated, poor blood flow \\
\hline
\end{tabular}

Table 2. Color Description of wound image 
Signal \& Image Processing : An International Journal (SIPIJ) Vol.5, No.2, April 2014

\subsection{Segmentation and Classification Techniques}

There are various segmentation techniques which helps in analyzing effective region of interest in wound images to acquire boundaries of a wound. After segmenting the wound from the wound image, segmented wound is classified based on the severity of the wound which can be identified by color histogram of the wound through color image processing techniques.

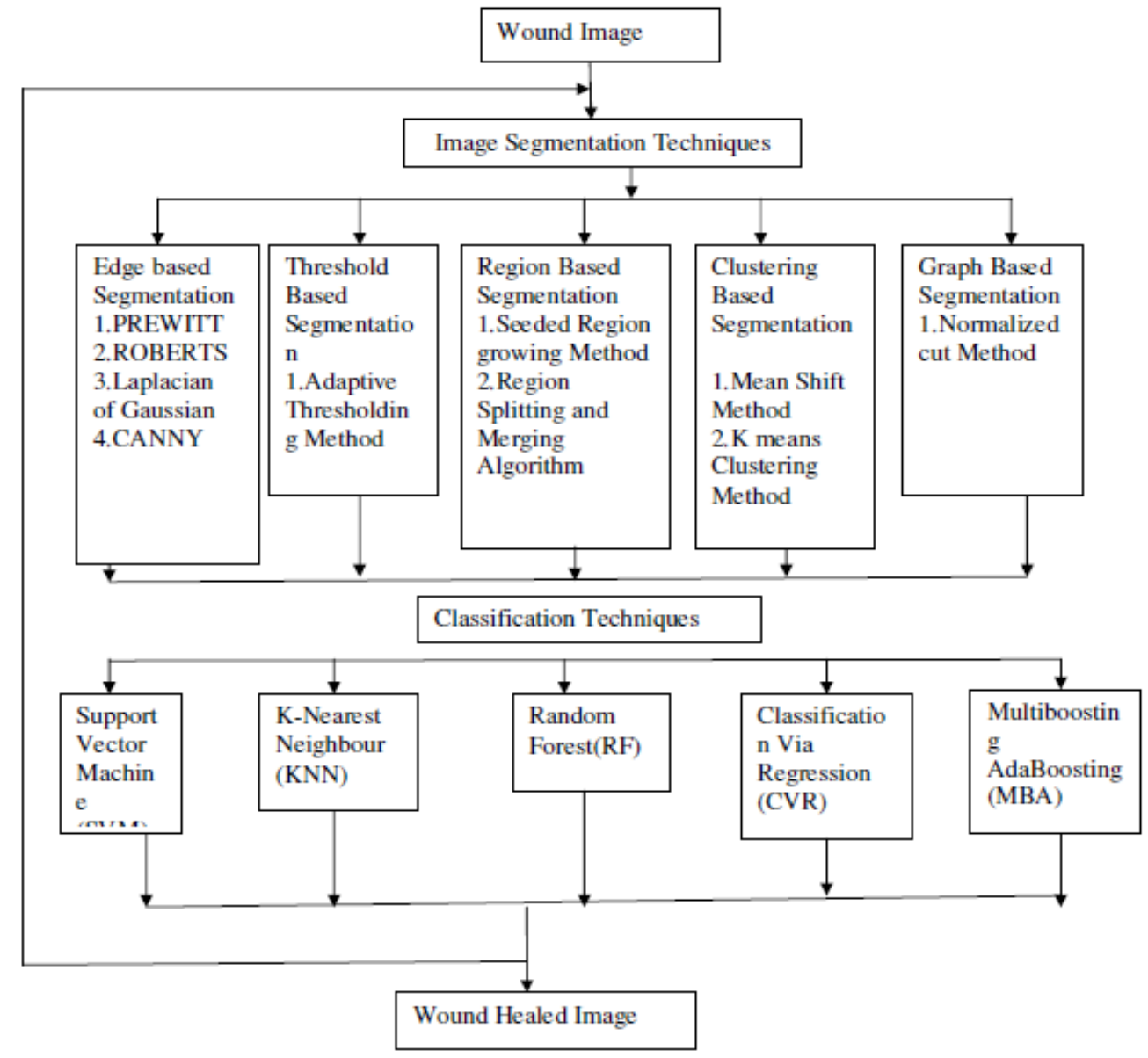

Fig 1: The flow chart for the proposed method

\section{WOUND IMAge ANALYSIS PROCESS}

The objective of our work is to develop an interactive tool for accurate assessment of the healing of the wound. This is done interactively with the help of inputs from the operator. This makes sure that the measurements take care of the wide variations and provides accurate results. A Wound can be identified by extracting boundaries of a wound by segmenting technique. Using preprocessing stage of a wound image quality can be increased by filtering and denoising techniques. Transparent layers have been applied to wound which is treatment for the wound and changes in appearance of the wound indicates healing status of a wound. 
Signal \& Image Processing : An International Journal (SIPIJ) Vol.5, No.2, April 2014

WIAC Algorithm:

1. Select a wound image from wound database acquired from open source wound images.

2. Segment the wound from the wound image using an efficient segmentation technique.

3. Improve the quality of the segmented wound from the wound image by using filtering, denoising techniques.

4. After preprocessing of segmented wound image overlay transparent layers of segmented wound shapes to reduce the intensity of colors in the wound.

5. Repeat step4 various times to get healed image of the wound.

6. Classify the segmented wound image into three labels based on severity level 0,1,2.

7. Conversion of segmented wound images from higher severity level to lower severity level leads to analyzing the healing status of the wound.

WIAC is used to classify the wounds based on color descriptors of various colors involves in analyzing the severity of the wound. WIAC framework is used effectively to forecast the healing process of a wound effectively which uses non contact method of analyzing the status of the wound. The transparent layers involved in reduction of severity of the wound which automates the healing process by using image overlay technique. This classifier also makes the resegmentation process by replacing the segmented wound with healed wound after undergoing various transparent layers. The below flowchart describes briefly the internal flow of WIAC.

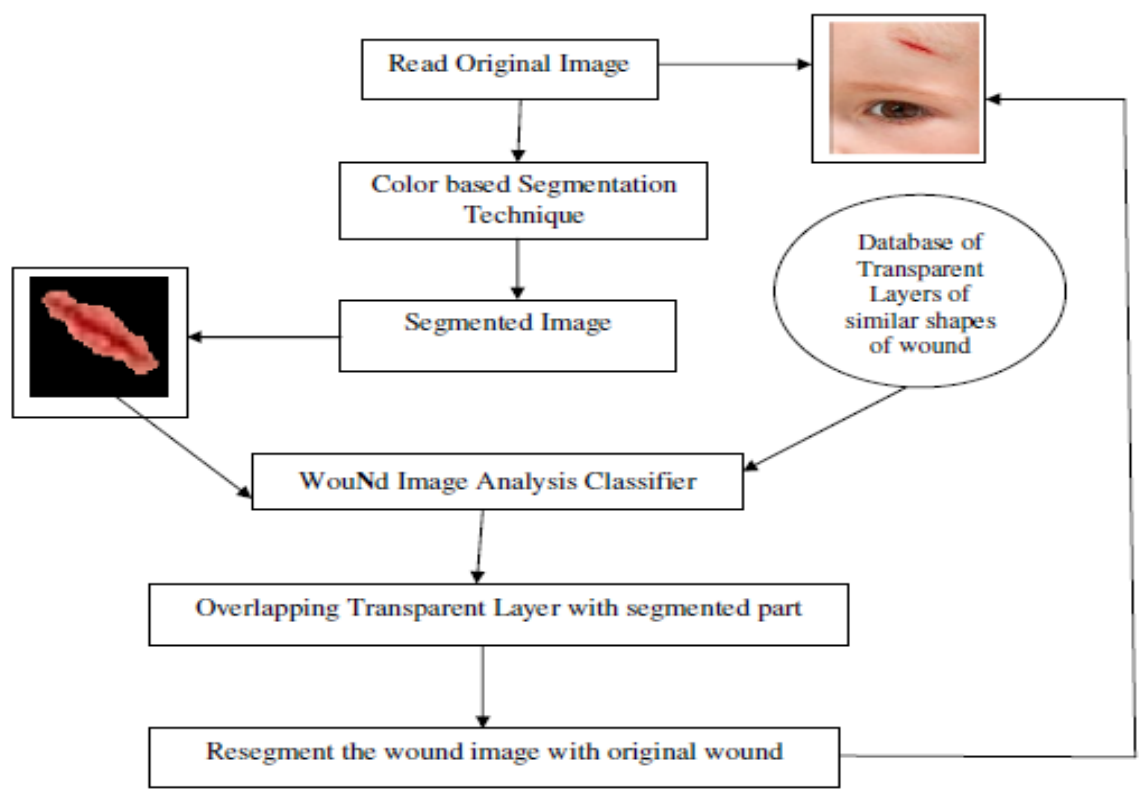

Fig 2 Flowchart for Wound Image Analysis Classifier(WIAC)

\section{EXPERIMENTAL RESULTS}

Wound Image Analysis classifier has been implemented using MATLAB. This classifier classifies the wound by considering healing status of the wound considering various colors involved in formation of wound and various stages undergoes by wound in healing process. Collection of wound image database from the open source wound images. The Fig4.1 illustrates 
Signal \& Image Processing : An International Journal (SIPIJ) Vol.5, No.2, April 2014

the collection of 50 images of various sizes. These images undergo segmentation technique to extract region of interest in the wound image which are depicted in the table 4.1. The segmented wound image undergoes filtering or denoising to remove the noise from the wound image. By considering the size and shape of the wound transparent layers are developed dynamically with respect to wound area which has been shown in Fig 4.2. The transparent layers of the wound image are overlayed and resultant wound image with decreased intensity of colors are obtained. As the area of the wound reduces indicates the healing of the wound. Texture measurement of the wound and after applying the overlay transparent layers on the wound indicates resultant wound image with reduce in size and shape of the wound. Tracking of wound healing by applying transparent layers are depicted in Fig4.3.

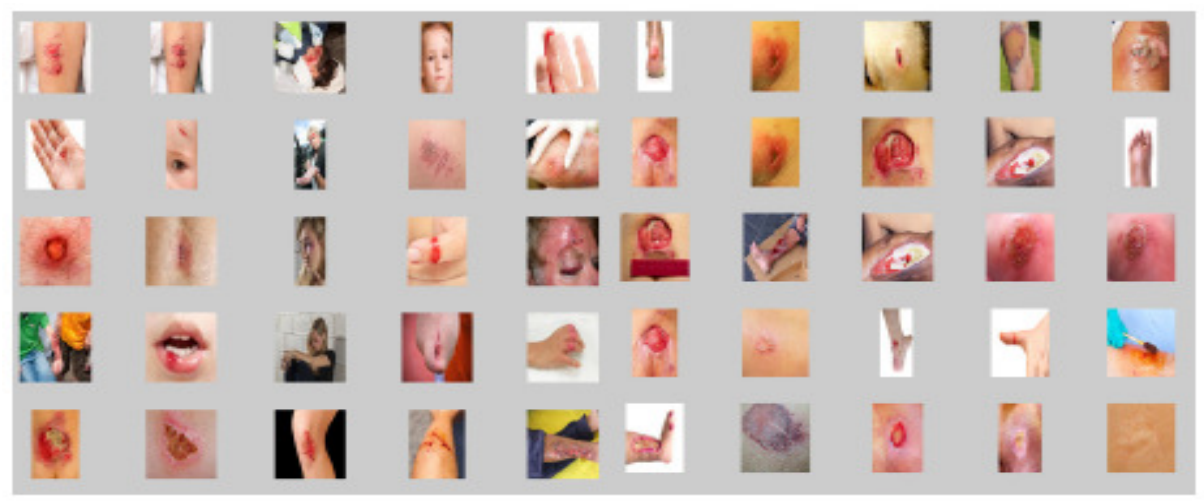

Fig 3: wound images collected from open source of wound database

Segmentation of the wound from the wound image involves in projecting the needed information by identifying the region of interest. After segmentation of the wound from the wound image, the wound undergoes formation of dynamic transparent layer and overlapping of segmented wound image with transparent layer undergoes changes in segmented wound image. After continuous repetition of the segmented wound by overlapping the dynamic transparent layer shown in Fig 4.2 and Fig 4.3

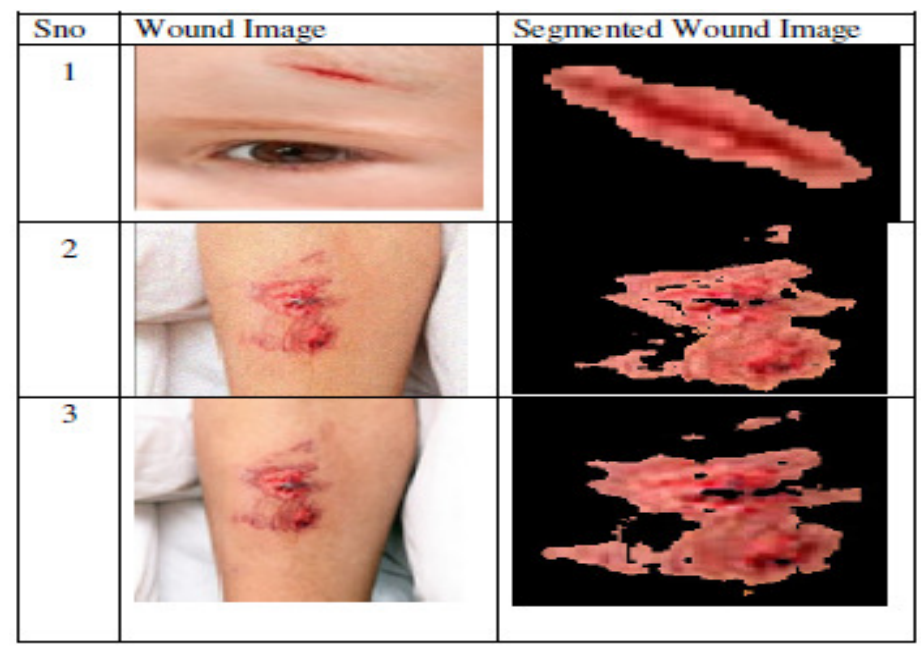

Table 3: Segmented Wound Images 
Signal \& Image Processing : An International Journal (SIPIJ) Vol.5, No.2, April 2014

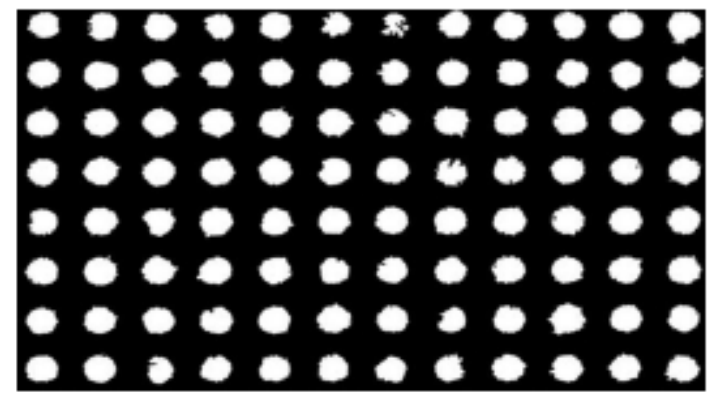

Fig 4: Transparent layers of various shapes of wound image

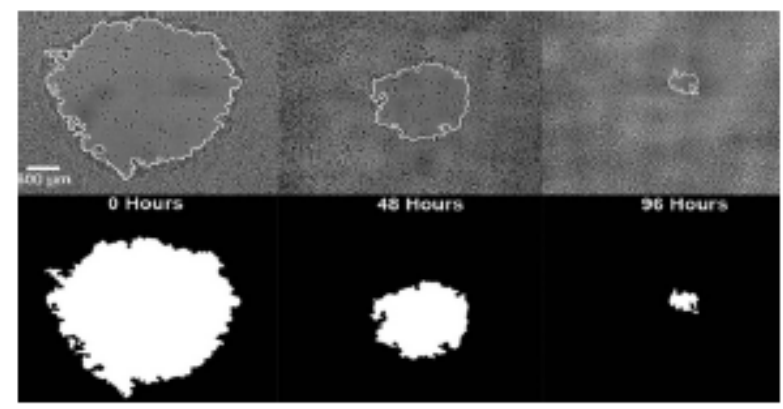

Fig 5: Tracking of wound healing by transparent layer

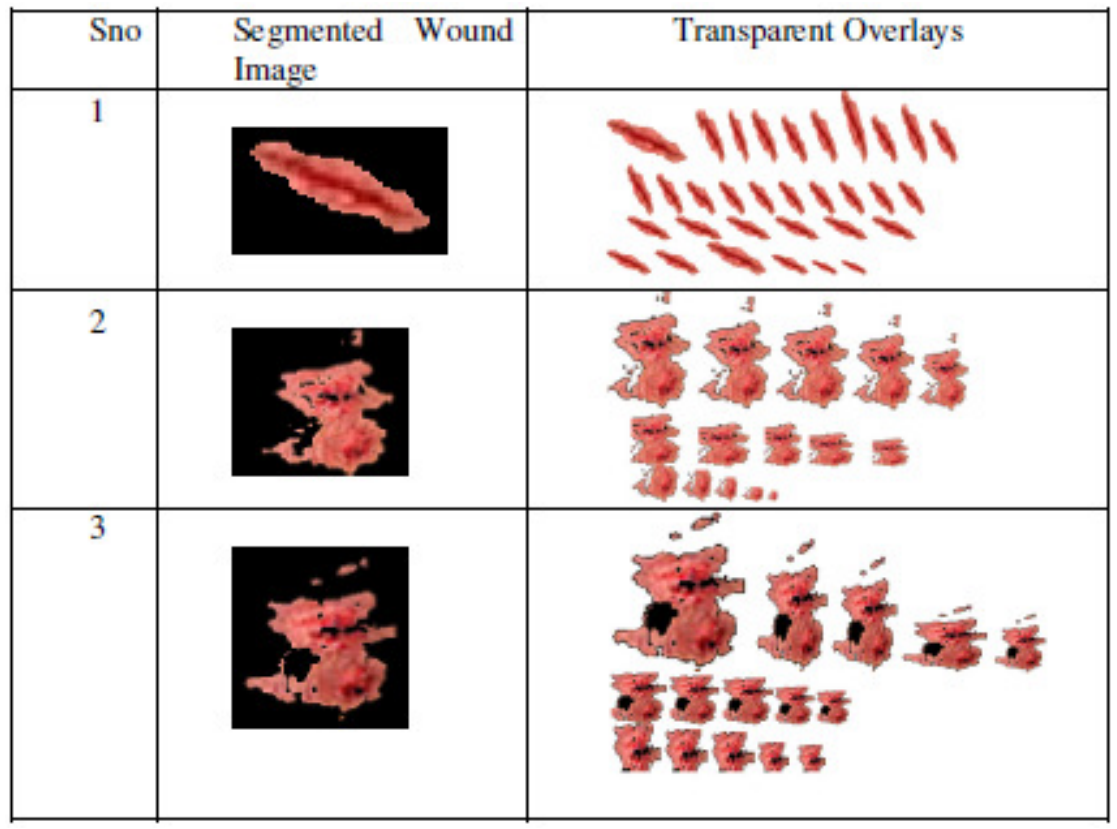

Table 4: Transparent overlays of segmented wound image 
Signal \& Image Processing : An International Journal (SIPIJ) Vol.5, No.2, April 2014

\section{CONCLUSIONS}

This paper concludes with developing a Wound Image Analysis Classifier (WIAC) for efficient tracking of wound healing status. Quantitative analysis of Wound healing status by developing an efficient classifier. Classifiers are used to classify wound images based on severity level of the wounds as labels. An effective tool has been developed for analysis of wound healing process using segmentation technique, filtering, denoising and transparent overlay techniques using color image processing. Effective detection of cells and estimation of their level of healthyness in the wound image and helping in diagnosing the status of wound in non contact manner to a doctor. Deterioration of wound can be easily analyzed using this tool. The images explored using this tool must be compared with real world wound image database and effectiveness can be calculated.

\section{REFERENCES}

[1] Kerstein MD: The scientific basis of healing. Adv Wound Care 1997; 10(3):30-36

[2] Kane D: Chronic wound healing and chronic wound management, in Krasner D, Rodeheaver GT, Sibbald RG. (eds): Chronic Wound Care: A Clinical Source Book for Healthcare Professionals, Third Edition. Wayne, PA, Health Management Publications, 2001,pp 7-17.

[3] B. Coulomb, P. Saiag, E. Bell, F. Breitburd, C. Lebreton, M. Heslan, and L. Dubertret, "A new method for studying epidermalization in vitro,” Brit. J. Dermatol., vol. 114, pp. 91-101, 1986.

[4] MacLeod J (ed): Davidson's Principles and Practice of Medicine, Thirteenth Edition. Edinburgh UK, 1981, pp 590-592.

[5] J. R. Mekkes and W. Westerhof, "Image processing in the study of wound healing," Clin. Dermatology, vol. 13, no. 4, pp. 401-407, 1995.

[6] Thomas A. Krouskop, Robert Baker, Michael S. Wilson, "A noncontact wound measurement system”,Journal of Rehabilitation Research and Development, Vol. 39 No. 3, Pages 337-346,2002.

[7] William Paul Berriss, Stephen John Sangwine, "Automatic Quantitative Analysis of Healing Skin Wounds using Colour Digital Image Processing”,World Wide Wounds ,Edition 1.1,1997.

[8] Herbin M, Bon FX, Venot A, Jenlouis F, Dubertret ML. "Assessment of healing kinetics through true color image processing", IEEE Transactions on Medical Imaging, 12(1). p. 39-43, Mar 1993.

[9] Thomas Gilman,"wound outcomes: the utility of surface measures",Lower Extremity wounds, 3(3),pp 125-132,2004.

[10] P Plassmann, KG Harding, JM Melhuish, "Methods of Measuring Wound size - A Comparative Study", WOUNDS, vol.6, no.2, p.54-61, 1994.

[11] David H. Keast, C. Keith Bowering etal, "MEASURE: A proposed assessment framework for developing best practice recommendations for wound assessment", Wound Repair and Regeneration, Volume 12 Issue s1 Page 1, 2004.

[12] Mark E.Roberts and Ela Claridge, "An artificially evolved vision system for segmenting skin lesion images”,MICCAI (1),pp 655-662,2003

[13] Julien Mairal, Michael Elad, and Guillermo Sapiro, "Sparse Representation for Color Image Restoration" IEEE Transactions On Image Processing, Vol. 17,No. 1,January 2008.

[14] Sylvie treuillent, Benjamin Albouy, and Yves Lucas "Three-Dimensional Assessment of Skin Wounds Using a Standard Digital Camera" IEEE Transactions On Medical Imaging, Vol. 28, No. 5, May 2009.

[15] Hazem Wannous, Yves Lucas, and Sylvie Treuillet "Enhanced Assessment of the WoundHealing Process by Accurate Multiview Tissue Classification "IEEE Transactions On Medical Imaging, Vol. 30, No. 2, February 2011

[16] Hazem Wannous a, Yves Lucas b, Sylvie Treuillet, "Efficient SVM classifier based on color and texture region features for wound tissue images", Medical Imaging 2008: Computer- Aided Diagnosis, Proc. of SPIE Vol. 6915, 69152T, (2008) • 
Signal \& Image Processing : An International Journal (SIPIJ) Vol.5, No.2, April 2014

\section{AUTHORS}

K Sundeep Kumar received the M.Tech (IT) from Punjabi University in 2003, ME (CSE) from Anna University in 2009 and pursuing Ph. D (CSE) from JNTUA. He is with the department of Information Science \& Engineering and as an Associate Professor, SEA College of Engineering and Technology, Bangalore. He has more than 25 Publications in various International Journals. His research interests include Image Processing, OOMD, Software Engineering and Data Warehousing. He is a life member in ISTE.

Dr. B. Eswara Reddy Graduated in B.Tech (CSE) from Sri Krishna Devaraya University in 1995. He received Masters Degree in M.Tech (Software Engineering), from JNT University, Hyderabad, in 1999. He received Ph.D in Computer Science \& Engineering from JNT University, Hyderabad, in 2008. He served as Assistant Professor from 1996 to 2006, Associate professor from 2006 to 2012.He served as Head of the Dept. of CSE during 2010 to 2012. Presently, he has been serving as professor of CSE Dept and Coordinator for Master of Science in Information Technology (MSIT)

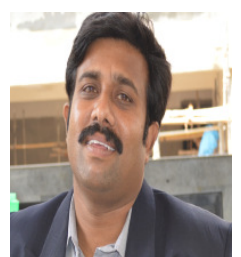
program, JNTUA in collaboration with CIHL.

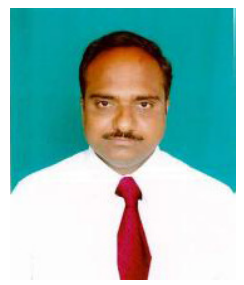

He has more than 30 Publications in various International Journals and more than 20 Publications in various National and International Conferences. He has authored two books: "Programming with Java published by Pearson/Sanguine Publishers" and "Data Mining: Principles and approaches" published. He has received University Grants Commission-Major Research Project(UGC-MRP) titled 'Cloud computing framework for rural health care in Indian scenario' His research interests include Cloud Computing, Pattern Recognition \& Image Analysis, Data Warehousing \& Mining and Software Engineering. He is a life member of ISTE, IE, ISCA, IAENG and member of CSI, IEEE. 